\title{
VIRTUAL PARADE GAME FOR LEAN TEACHING AND LEARNING IN STUDENTS FROM BRAZIL AND CHILE
}

\author{
Clarissa N. Biotto ${ }^{1}$, Rodrigo F. Herrera ${ }^{2}$, Luis A. Salazar ${ }^{3}$, Cristina T. Pérez $^{4}$, \\ Roberto M. Luna ${ }^{5}$, Priscila M. Rodrigheri ${ }^{6}$, and Sheyla M. B. Serra ${ }^{7}$
}

\begin{abstract}
The use of games in engineering teaching is common practice in classes with lecturers all over the world. However, due to the COVID-19 pandemic, undergraduate civil engineering education became virtual and remote. In this context, many games traditionally played in person among students have undergone adaptations to the digital environment. The game "Parade of Trades" or "Parade Game" is used worldwide to teach the effects of variability in construction workflows in linear, dependent and sequential production systems. An adapted version of the game to the virtual environment was proposed by ASKM \& Associates LLC and Navilean LLC. It was presented at the International Group for Lean Construction Congress (IGLC 2020). This version of the Parade Game was applied in three different high education institutions in Brazil and Chile. The game's effectiveness for teaching the variability concept was tested by administering a questionnaire before and after the game with the Production Planning and Control course's students in Civil Engineering. The main contribution of this study is the evaluation of learning brought by the game. Results show an increase of $20 \%$ in the correct answers in the post-game questionnaire, demonstrating that the students captured the game's main concepts.
\end{abstract}

\section{KEYWORDS}

Engineering education, lean games, parade game, COVID-19, virtual teaching.

1 Post-Doctoral Researcher, Postgraduate Programme of Civil Engineering, Federal University of Sao Carlos (UFSCar), Brazil, clarissa.biotto@ufscar.br, orcid.org/0000-0002-2433-6735

2 Professor, School of Civil Engineering, Pontificia Universidad Católica de Valparaíso, Chile, rodrigo.herrera@pucv.cl, orcid.org/0000-0001-5186-3154

3 PhD Candidate, Department of Construction Engineering and Management, Pontificia Universidad Católica de Chile, and Assistant Professor, Faculty of Engineering, Universidad Andres Bello, Chile, lasalaza@uc.cl, orcid.org/0000-0001-7339-8935

4 Post-Doctoral Researcher, Department of Civil and Architectural Engineering, Aarhus University, Denmark, cristina.toca.perez@cae.au.dk. orcid.org/0000-0002-4182-1492

$5 \mathrm{PhD}$ Candidate, Department of Construction Engineering and Management, Pontificia Universidad Católica de Chile, raluna@uc.cl, orcid.org/0000-0001-7647-7049

6 Master Candidate, Postgraduate Programme of Civil Engineering, Federal University of Sao Carlos, Brazil, eng.priscilamr@ gmail.com, orcid.org/0000-0002-6067-8943

7 Professor, Civil Engineering Department, Federal University of Sao Carlos, Brazil, sheylabs@ufscar.br, orcid.org/0000-0002-9508-976X 


\section{INTRODUCTION}

The "Parade of Trades" or "Parade Game" consists of a game to demonstrate the impacts of variability on a construction project, in which multiple teams are working independently, and often, sequential activities (Choo and Tommelein 1999). Playing this game teaches the concept of workflow reliability, where workflow is expressed by the number of units of work that get passed from one construction trade to the next. The game is based on a simple linear sequence of sequentially dependent process steps with handoffs from one stage to the next determined by the roll of a die, thus mimicking a process subject to variability (Tommelein et al. 1999; Tommelein et al. 1998).

Despite being initially a personal game simulation or a computer simulated-based game, the Parade Game can also be played physically in a classroom. The game lends itself to a rich discussion of strategies to cope with variability in production systems. Since the Parade of Trades was introduced as a teaching tool for Lean construction in the late 1990s, it has not only become a widely used exercise in classroom and practitioner training settings to teach Production Planning and Control concepts (Tommelein 2020), but also a reference system for further study by scholars worldwide (Deshpande and Huang 2011). At the onset of the crisis caused by COVID-19 in early 2020, the traditional educational model, based on masterclasses and linear teaching materials, required adaptation to the demands of society. As a result, it became necessary to use technology to address the challenges experienced by several universities, from traditional learning to emergency remote teaching (ERT), and the impact on apprenticeship and student satisfaction (Hodges et al. 2020).

In such circumstances, five researchers and academics from different institutions (Brazil, Chile, Ecuador, and Spain) decided to create the Research Group of Young Researchers for Architecture, Engineering, and Construction Industry (YR4 AECI www.yr4aeci.org) on May $29^{\text {th, }} 2020$.

One of those challenges was to find ways to adapt the Parade Game back to the virtual environment without using computer simulations. There are several reasons for not using the game with computer simulation, some examples proposed by Deshpande and Huang (2011) are (1) unawareness of the capabilities of simulation tools, (2) difficulty in obtaining the necessary resources (i.e., computers and simulation software), and (3) the instructor's inability to use the latest technology.

In this way, a version created by ASKM \& Associates, LLC and Navilean LLC under copyright Creative Commons license - Attribution-ShareAlike 4.0 International (CC BYSA 4.0) was presented at the $28^{\text {th }}$ Congress of the International Group for Lean Construction (IGLC 2020) during the lean construction gaming session. Furthermore, the template developed in MS Excel was made available to the simulation participants by the game facilitator.

This work aims to measure the students' learning of the variability concept using the Parade Game version developed by ASKM \& Associates. For that, the authors compared the results of the game application in three different universities of South America, those being: Universidade Federal de São Carlos (UFSCar), Universidad Nacional Andrés Bello (UNAB) and Pontificia Universidad Católica de Valparaíso (PUCV). 


\section{BACKGROUND}

\section{Origin of Parade of Trades Game}

Initially, Greg Howell created the game to teach construction students at the University of New Mexico in early 1994. In 1998, the Parade Game was developed to research lean construction and new materials management technologies (Tommelein et al. 1999). In this version, the game takes place in a computing environment using the dice game strategy and the software STROBOSCOPE, an acronym for STate and ResOurce Based Simulation of COnstruction ProcEsses. Stroboscope is a simulation programming language specifically designed to model construction operations of any complexity (Martinez 1996). Alarcón and Ashley (1999) also developed a Parade Game version using the simulation program @ Risk.

The Lean Construction Institute (2020) markets a game kit containing seven sets of 35 colored chips; 7 dice sets - each pack has ten dice in total: 3 reds, three blues, three blacks, one green; 49 spreadsheets; 7 scoresheets; 1 instruction manual; 1 Parade of Trades in CD-Room. The computer simulation allows students to experiment with various alternatives to sharpen their intuition regarding variability, process yield, buffers, productivity, and team sizing (Tommelein et al. 1999).

\section{The New Version of Parade of Trades}

The game consists of the execution of seven activities, linear and sequential, that must occur on each floor of a 35-story building. The game has the following objectives: (1) to understand the effect of process variability on the workflow of dependent activities, distinguishing the production capacity of the teams; (2) to understand what buffers are and what they are for; and (3) to interpret a flowline of the game's activities. The definitions of the concepts used in the game are:

- Production capacity: the number of activities per unit of time that a crew is technically able to finalize if there are no constraints (Tommelein et al. 1999).

- Production rate: number of activities per unit of time a team can perform when considering different constraints such as lack of material, incomplete predecessor activity, or wrong place for work (Tommelein et al. 1999).

- Buffers: Strategy to protect dependent activities to ensure their execution as planned (i.e., by making the crew have service packages available to execute so that the constraints do not influence the actual production) (Koskela 2000; Tommelein et al. 1999).

- Flowline chart: graphical representations of the number of service packages performed as a function of time, making it possible to verify the location of the services performed, identify the cycle, waiting time, and variability, making the process transparent (Priven et al. 2014; Tommelein et al. 1999).

The game is played in two rounds to understand the concepts presented; each one is available in the template tabs, and the results obtained in them must be compared with the steady production tab. The game is based on dice probabilities, using cubic dice with six faces. Figure 1 illustrates the dynamics of the game.

The game requires eight people: one is the facilitator who leads the game, and the other seven are the crew' leaders of each activity. The last activity, Accessories, will only start in the seventh week, namely, in the game's seventh round. Each activity's production capacity in each round is defined by the number displayed in the dice rolled by the crew's 
leader of that activity. Thus, the maximum production capacity of a team is six floors per week (round), and the minimum is one floor per week; consequently, the average productivity of each crew is 3.5 floors per week. The average productivity for the seven activities on the 35 floors of the building is determined after concluded the first activity, "Layout", will be completed on all 35 floors in the $10^{\text {th }}$ week, and the last activity, "Accessories", will be completed in the $16^{\text {th }}$ week.

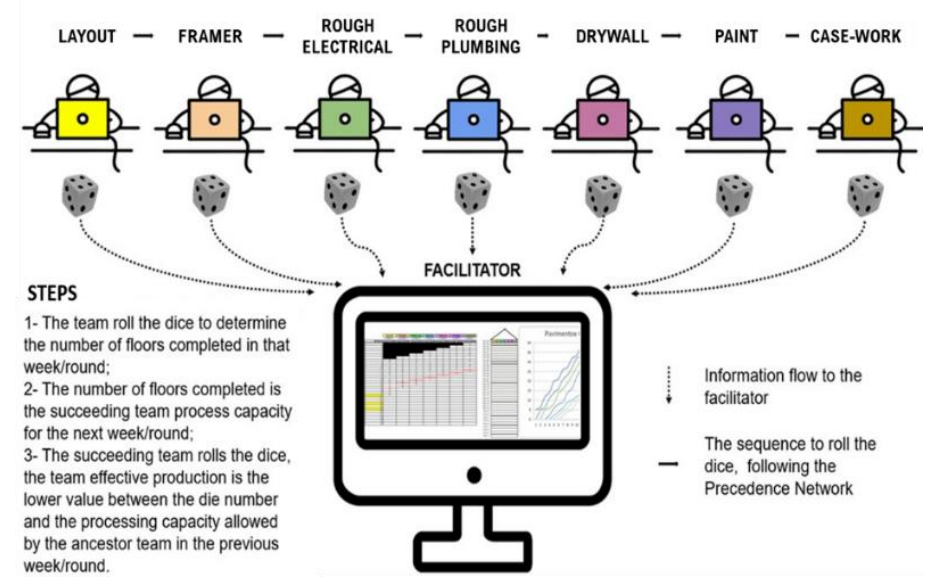

Figure 1: Parade Game version adopted in this work

Let us consider that all activities achieve the maximum production capacity (six floors per week). The first activity will be completed in the $6^{\text {th }}$ week and the last activity in the $12^{\text {th }}$ week. If we consider the minimum production capacity ( 1 floor per week), the building's deadline extends to 41 weeks. Since the proposed game is based on the chances that six-sided cubic dice can result in each roll, the building's deadline should be between 12 to 41 weeks.

For the first seven rounds, one activity is included at a time, so only the "Layout" team rolls the dice in the first round, resulting in productivity for the week. Then, in the second round, the "Framer" team starts rolling the dice to result in this team's production capacity for the week. However, at this time, the production of the predecessor team ("Layout") in the previous week should be considered to identify whether the production capacity might be reached or not.

In the first seven weeks, in each round, a new activity or team is included in the game, and its production capacity for the week is defined by the number displayed on the die. As crews execute the possible production in each round/week, the 35 floors of the building will be progressing until all the seven teams arrive at the last floor, i.e., when the game ends. At this moment, it is possible to (a) draw the flowlines chart according to the productivity executed by the crews every week; (b) interpret and analyze the impact of variability in crews' productivity in successor activities; and (c) discuss the use of buffers to protect the production against variability. The final discussion with the students is essential to achieve the learning objectives proposed by the game.

\section{RESEARCH METHOD}

The main activities conducted were: (1) population selection for applying the game; (2) questionnaire development; (3) game application; and (4) game results analysis. 


\section{Population Selection for ApPlying The GAMe}

The game was played by three student groups in three universities, one from Brazil and two from Chile. The main characteristics of each course and the game participants are summarized in Table 1.

Table 1: Description of the courses and game participants

\begin{tabular}{|c|c|c|c|}
\hline & $\begin{array}{l}\text { Universidade Federal } \\
\text { São Carlos (UFSCar) }\end{array}$ & $\begin{array}{c}\text { Pontificia Universidad } \\
\text { Católica de Valparaíso } \\
\text { (PUCV) }\end{array}$ & $\begin{array}{l}\text { Andres Bello } \\
\text { University } \\
\text { (UNAB) }\end{array}$ \\
\hline Bachelor course & Civil Engineering & Civil Engineering & Civil Engineering \\
\hline Name of the course & $\begin{array}{c}\text { Planning and Control of } \\
\text { Production }\end{array}$ & $\begin{array}{c}\text { Planning and Control of } \\
\text { Projects }\end{array}$ & Lean Construction \\
\hline Application day & September 3rd 2020 & October 14th 2020 & October 15th 2020 \\
\hline Platform & Google Meet Platform & Zoom & BlackBoard \\
\hline Number of students & 60 & 23 & 7 \\
\hline $\begin{array}{l}\text { Number of } \\
\text { responses }\end{array}$ & 54 & 22 & 7 \\
\hline
\end{tabular}

Due to the COVID-19 pandemic, classes were offered remotely on an emergency basis and not mandatory. We collected 83 responses from this questionnaire from a total of 90 participating students. Students do not know the correct answers.

\section{QUESTIONNAIRE DEVELOPMENT}

The task of creating the questionnaire involved three main steps. Firstly, two of the authors of this paper developed a pilot questionnaire. For that, they determined the questionnaire format, the questionnaire length, and the concepts to evaluate. Then, an expert committee made by the rest of the authors of this paper ( 5 members) reviewed the questionnaire to make sure it was accurate, free of item construction problems, and grammatically correct. In addition, during the questionnaire evaluation, the committee analyzed the validity of the constructs, those being: process variability, buffers, and flowline. The construct validity can be evaluated by estimating its association with other variables (or measures of a construct). Furthermore, in this study, for understanding the constructs, several concepts were adopted (production capacity and production rate), as previously mentioned. Finally, the final version of the questionnaire (Table 2) was developed, and the correct answers were highlighted in bold.

Table 2: Questionnaire developed for evaluating the learning of the concept of variability through the game Parade of Trades

\begin{tabular}{ll}
\hline $\mathbf{N}^{\circ}$ & \multicolumn{1}{c}{ Questions and alternatives } \\
\hline $\mathbf{1}$ & What effects of high variability (in construction processes with dependency relationships) on a \\
construction project? & a: Increases productivity, decreases runtime, and improves performance. \\
b: Decreased productivity, increases runtime, and reduces performance. \\
c: None of the above. \\
d: All previous ones. \\
e: I don't know the answer. \\
$\mathbf{2}$ What is the difference between processing capacity and production rate? \\
a: There is no difference between the two concepts. \\
b: Processing capacity is always greater than or equal to the production rate. \\
c: Production rate can be greater than processing capacity. \\
d: Production rate can never be achieved. \\
e: I don't know the answer.
\end{tabular}




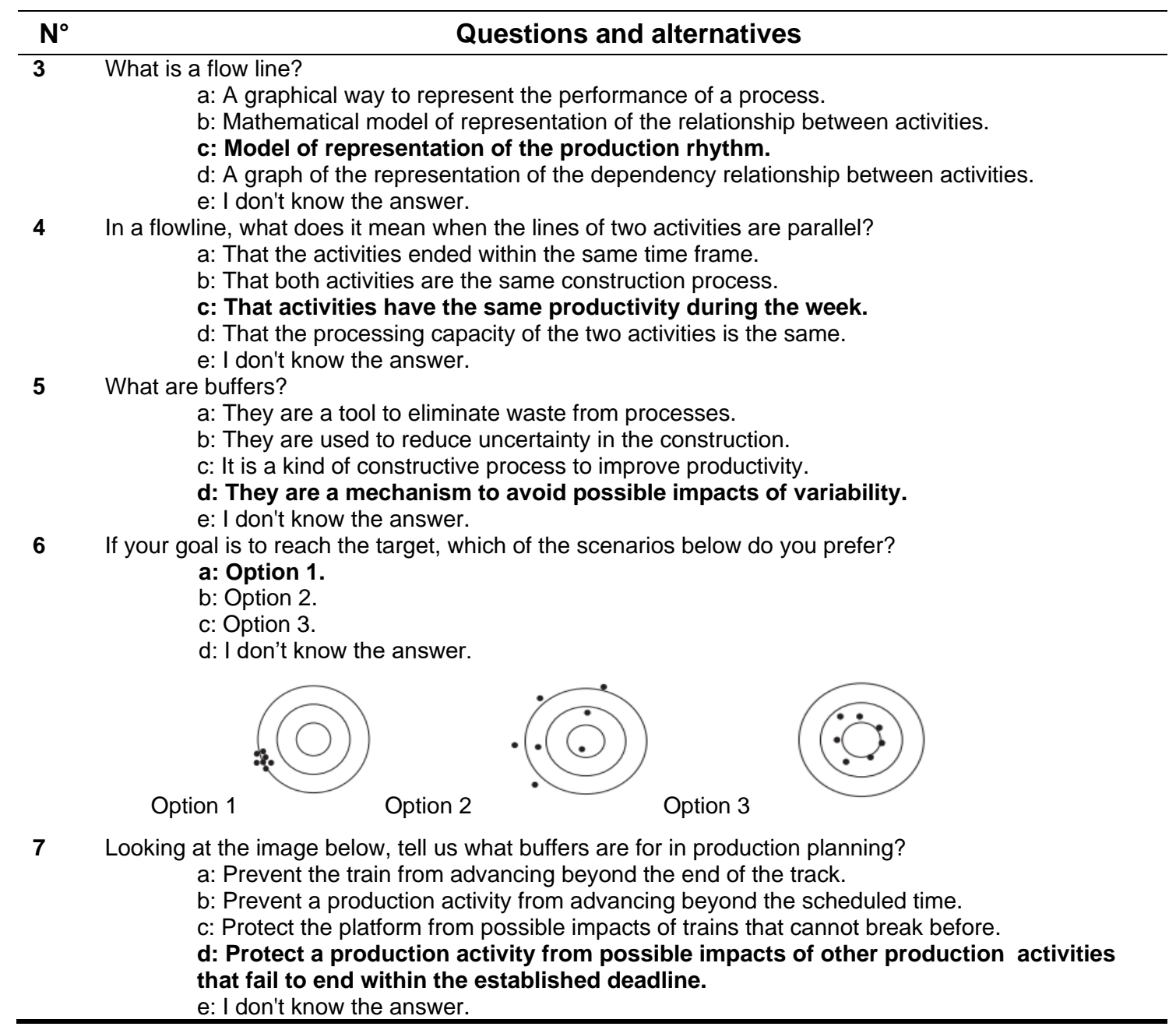

\section{GAME APPLICATION}

The game application involved four different phases: (1) Pre-game questionnaire: Access and answer to the questionnaire; (2) Game presentation to students: play the game with volunteers; (3) Play the game with all the students (volunteers and facilitators); and (4) Post-game questionnaire: re-answer the same questions.

In the first phase, the students received the access link to a virtual questionnaire composed of seven multiple-choice questions, including "I don't know the answer" as one of the answer alternatives.

In the second phase, the game was presented to the students by sharing the teacher's screen, and its objectives and rules were described. The first game round was developed with the teacher as a facilitator and volunteer students as team leaders of the seven activities depicted in the network of Figure 1. The facilitator collected the crews' productivity information and typed in the template available in MS Excel. It was possible to follow the activities' progress on the building's different floors in each round (Figure 2). As described above, students representing each activity reported the number obtained by throwing the virtual dice (a designated website was used). The first-round results were represented in the flowline graph of Figure 3b, and the variability and buffers concepts were explored with the students.

In the third phase, the first volunteer students from the first round became facilitators of a new round with other students. They used a virtual meeting platform to create new 
meeting rooms and invited seven students to join them. After the completion of this new match, the graphs of the groups of students that reached the shortest and longest lead time of the building construction were compared and discussed again, observing the characteristics of the steady production flowlines (Figure 3a), and, again, reinforcing the concepts of variability and buffers.

The fourth and final phase was dedicated to re-answer the questionnaire presented in Table 2 to measure the learning brought after the game.
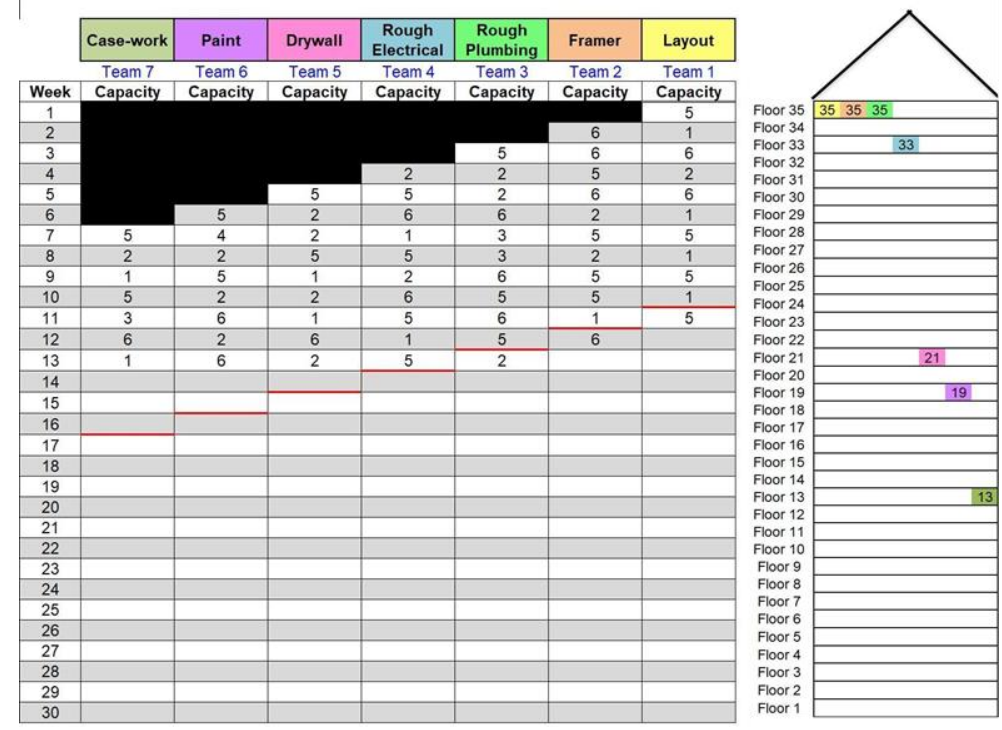

Figure 2: Game scenario (first-round): week 13 of the building process. Teams 4 to 7 have not yet finished their tasks.
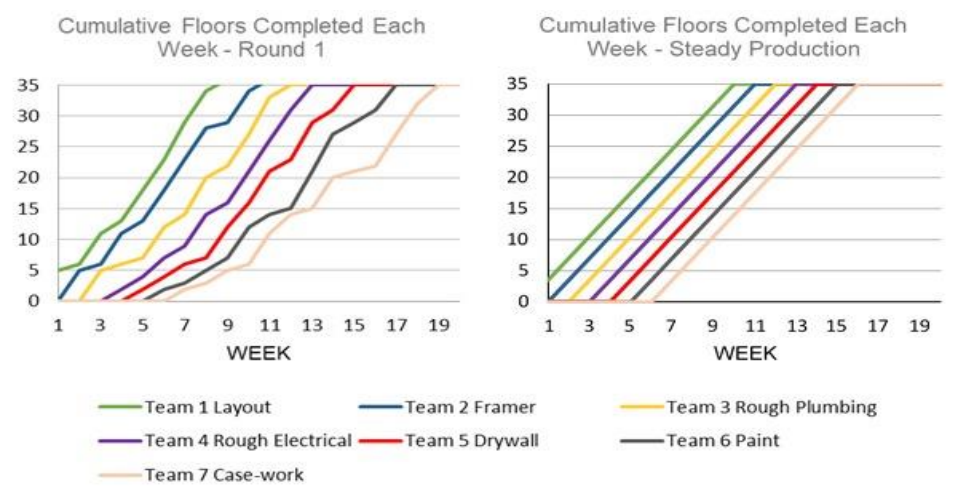

Team 3 Rough Plumbing

Figure 3: Graphs of flowlines of (a) performed by the teacher-facilitated, and (b) steady production

\section{GAME ReSUlts ANALYSIS}

At the end of the game, the students answered two additional questions. Firstly, the students answered the following question "Do you believe that this sort of game strengthens the process of teaching-learning?" For this question, the Likert Scale of five points was adopted: (1) strongly disapprove; (2) disapprove; (3) neutral; (4) approve; and (5) strongly approve. Secondly, the students answered the closed question: "Would you like to play other games like today's game in the future?"

Finally, a reflection was made comparing the initial and final results, analyzing the correct answers and the most common errors. The analysis consisted of calculating, in 
each question, the percentage of students who answered correctly before and after the game. Additionally, in each question, the percentage of students who did not know the answer before and after the game was calculated.

\section{RESULTS AND DISCUSSION}

We analyzed the pre-and-post-game responses provided in the questionnaire. Figure 4 presents the percentage of correct answers per question before and after the game application. Also, Figure 5 shows the percentage of students who marked the alternative "I don't know the answer" before and after the game. In general, there was an increase of $20 \%$ in the correct answers in the post-game scenario and a decrease of $12 \%$ in the "I don't know" answer, therefore reinforcing that virtual educational games are an essential learning tool. The following is a question-by-question analysis.

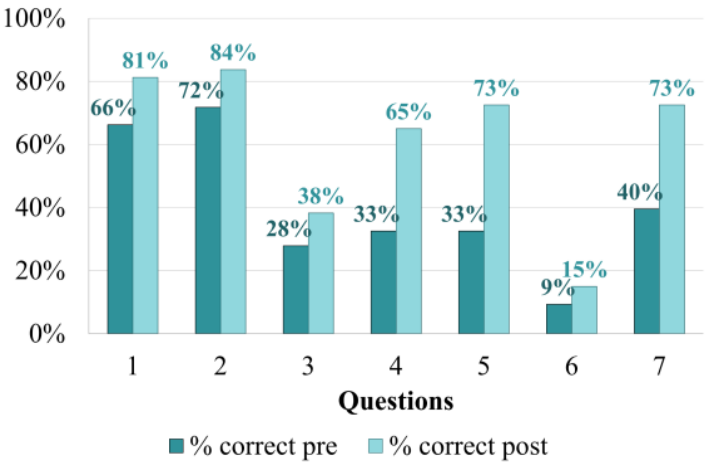

Figure 4: Percentage of students' correct answers to the pre-and-post-game questionnaire

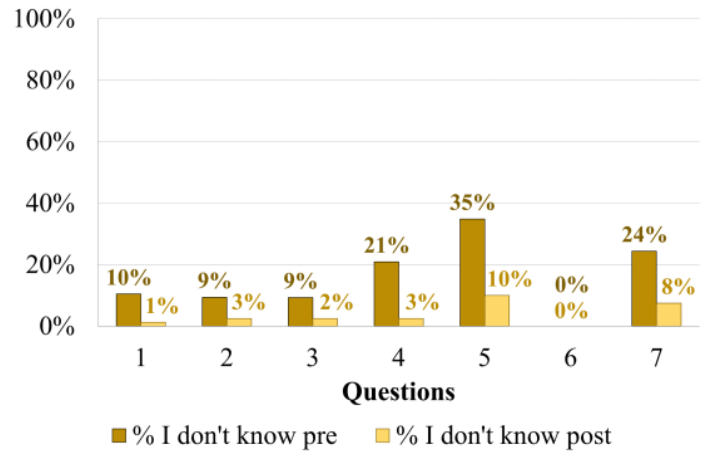

Figure 5: Percentage of students' "I don't know the answer" to the pre-and-post-game questionnaire

First, question 1, related to the impact of the variability in the construction process, has a high percentage of correct answers, even in the pre-game scenario $(66 \%$ of correct answers, Figure 4, i.e., most of the civil engineering students sensed the impact of variability before playing the game). Then, post-game, this percentage rises to $81 \%$ (Figure 4) of correct answers, while the percentage of students who responded that they did not know the answer decreased by $9 \%$ (from $10 \%$ to $1 \%$, Figure 5 ). Therefore, it is possible to infer that most of the increase in correct answers is associated with students who were not sure of the impact of variability before the game was played.

Second, question 2, related to the difference between the processing capacity and production rate, has a high percentage of correct answers in the pre-game scenario $(72 \%$, Figure 4). After the game, the correct answers' portion increased to $84 \%$ (difference of $12 \%$, Figure 4 ), and the percentage of students who did not know the answer was only reduced by $6 \%$ (from $9 \%$ to 6\%, Figure 5). In post-game, a group of students who did not know the answer succeeded in answering correctly, and a percentage of students who answered incorrectly in the pre-game succeeded in answering correctly after the activity.

Third, questions 4, 5, and 7 had the most significant correct answers from pre-game to post-game. Only about one-third of the students answered correctly in the pre-game, while two-thirds of the students got the correct answer in the post-game. Notably, in question 5, associated with buffers' concept, $35 \%$ of the students answered "I do not know the answer" in the pre-game, a percentage that was reduced to $10 \%$ in the post-game questionnaire (Figure 5). 
Finally, it is observed that, for questions 3 and 6, less than half of the students selected the correct alternative even after the game (38\% and $15 \%$ of correct answers, respectively, Figure 4). In this context, it is also observed that question 3 had a high frequency of "I do not know the answer" alternative, even after the game application (9\%, Figure 5). These observations indicate that the knowledge necessary to answer such questions correctly was not sufficiently explored by the game for most students, despite the knowledge gained in the pre-and-post-game stages. Mainly, question 6, related to the concepts of precision and accuracy, is the one with the lowest percentage of correct answers (9\% in the pre-game and $15 \%$ in the post-game, Figure 4); additionally, students did not doubt their answer, always preferring low precision (high variability) and high accuracy. These results reinforce the need for a paradigm change in production systems and variability in construction projects. Questions 3 and 6 explore, respectively, the concepts of flowline and how variability influences decision-making. These concepts were addressed after applying the game and should be put in focus in classes. Subsequently, most students can understand these concepts and apply them to production planning and control.

The results of the game evaluation to measure the level of approval from "strongly disapprove" to "strongly approve" indicate that most students consistently approve of the game. To summarize, $56 \%$ of responses strongly showed approval, $32 \%$ approve, $11.5 \%$ indecision, and $0 \%$ answers were strongly disapproving or disapproving. Regarding the students' interest in playing a similar game in the future, the yes-no question's results indicate that $87.5 \%$ of the students would like to play again, $2.5 \%$ of the students would not like to repeat, and $10 \%$ would like to play again of the students showed indecision.

\section{CONCLUSION}

This study aimed to evaluate the effectiveness of the "Parade of Trades" game for teaching the variability concept. A questionnaire was developed and tested with the Production Planning and Control course's students in Civil Engineering in three universities, one from Brazil and two from Chile. The main contribution of this study is the evaluation of learning brought by the game.

By administering the same questionnaire in two phases, before and after the game, it was possible to measure the students' learning about the concepts addressed by the game. Evaluating the pre-and-post-game responses, there was an increase of $20 \%$ of correct answers and a reduction of $9 \%$ in the alternative "I don't know the answer" in all questions. Besides, this work allows educators in engineering, architecture, and construction schools to use the game in virtual environments. The experience of applying the Parade of Trades game to the virtual environment proved successful, considering the students' engagement and the use of different tools that facilitate remote teaching.

This work's main limitations where the virtual game was applied in only two countries; therefore, it is recommended that the game and questionnaires be used in a larger sample of countries and cultures. In addition, in the three applications of the game, the educators did not explain previous concepts theoretically; therefore, it would be interesting to evaluate two scenarios (1) with previous theoretical explanation and (2) without an earlier theoretical explanation. The questionnaire's answers were anonymous, which did not allow individual traceability of the percentage of correct answers of each student before and after the game; therefore, it is recommended to conduct a personal analysis and evaluate if there are significant differences between the pre-game and postgame. The results of the application of the questionnaire showed a low percentage of achievement in the identification and comparison between the concepts "precision" and 
"accuracy" - question 6; therefore, it is necessary to review how to integrate these concepts in the game and check if the question related to this topic is the one allows demonstrating the understanding of these concepts.

\section{ACKNOWLEDGMENTS}

The authors would like to thank the ASKM \& Associates, LLC and Navilean LLC for sharing the template with the IGLC2020 participants; the YR4-AECI Researchers who played the game for improvements and created the questionnaire. The first author would like to thank CAPES, Brazil, for the PNPD postdoctoral scholarship.

\section{REFERENCES}

Alarcón, L.F., and Ashley, D.B. (1999). "Playing games: Evaluating the impact of lean production strategies on project cost and schedule." Proc. 7th Ann. Conf. Int. Group for Lean Construction, p.263-274, Berkeley, USA.

Choo, H. J., and Tommelein, I. D. (1999). "Space scheduling using flow analysis." Proc. 7th Ann. Conf. Int. Group for Lean Construction, p.299-312, Berkeley, USA.

Deshpande, A.A., and Huang, S. H. (2011). "Simulation games in engineering education: A state-of-the-art review." Computer Applications in Engineering Education, v.19, n.3, p.399-410. doi.org/10.1002/cae.20323.

Hodges, C., Moore, S., Lockee, B., Trust, T.; Bond, A. (2020). "The difference between emergency remote teaching and online learning." Educ. Rev. 27, v.3, p.1-12.

Koskela, L. (2000). "An exploration towards a production theory and its application to construction." 296 f. Doctoral thesis. Technical Research Centre of Finland, VTT Building Technology, Finland.

Lean Construction Institute (LCI). (2020). "Parade of trades simulation." https://www.leanconstruction.org/learning/game-simulations/parade-of-tradessimulation/.

Martinez, J.C. (1996). "STROBOSCOPE State and Resource Based Simulation of Construction Processes." Doctoral thesis, University of Michigan, Ann Arbor, Michigan. Available in: http://www.ioannou.org/stroboscope/strobo-pubs.

Priven, V., Sacks, R., Seppänen, O., and Savosnick, J. (2015). "A lean workflow index for construction projects." Proc. 22nd Ann. Conf. Int. Group for Lean Construction, p.715-726, Oslo, Norway.

Rybkowski, K.S., Forbes, L. H., and Tsao, C. (2018). "The evolution of lean construction education (Part 1 of 2): At US-based universities. Proc. 26th Ann. Conf. Int. Group for Lean Construction, p.1013-1023, Chennai, India.

Tommelein, I. D., Riley, D. R. and Howell, G. A. (1998), "Parade Game: Impact of Workflow Variability on Succeeding Trade Performance" Proc. 6th Ann. Conf. Int. Group for Lean Construction, p.13-15, Guarujá, Brazil.

Tommelein, I. D., Riley, D. R., and Howell, G. A. (1999). "Parade game: Impact of workflow variability on trade performance." J. Constr. Eng. Manag., v.125, n.5, p.304-310. doi.org/10.1061/(ASCE)0733-9364(1999)125:5(304).

Tommelein, I. D. (2020). "Takting the Parade of Trades: Use of Capacity Buffers to Gain Work Flow Reliability." Proc. 28th Ann. Conf. Int. Group for Lean Construction, p.421-432, Berkeley, USA. 